\section{IN BRIEF}

\section{$\Rightarrow$ REGULATORY T CELLS}

Toll-like receptor 2 signaling modulates the function of $\mathrm{CD} 4{ }^{+} \mathrm{CD} 25+$ regulatory $\mathrm{T}$ cells.

\section{Liu, H. et al. Proc. Natl Acad. Sci. USA 103, 7048-7053 (2006)}

Given that there are numerous regulatory T-cell populations that suppress T-cell responses, how does successful protective immunity occur in the face of such suppression? Foo Liew and colleagues report that, in a similar manner to effector T cells, naturally occurring regulatory $T\left(T_{\text {Reg }}\right)$ cells express Toll-like receptor 2 (TLR2) and undergo marked proliferation in the presence of the TLR2 ligand bacterial lipoprotein (BLP) and CD3specific antibody. Importantly, BLP-induced $\mathrm{T}_{\text {Reg }}$-cell proliferation resulted in a transient loss of suppressive activity and forkhead box P3 (FOXP3) expression. In addition, interleukin-2 produced by BLP-activated effector $T$ cells rendered these cells refractory to $T_{\text {e }}$-cell-mediated suppression. But, 3 days after BLPmediated activation, the $\mathrm{T}_{\text {Reg }}$ cells regained FOXP3 expression and suppressive activity, as indicated by their ability, following adoptive transfer, to prevent colitis in immunodeficient mice.

\section{$\Rightarrow$ ANTIGEN-PRESENTING CELLS}

$\mathrm{CD}_{1} \mathrm{a}^{+}$antigen-presenting cells in human dermis respond rapidly to CCR7 ligands.

Angel, C. E. et al. J. Immunol. 176, 5730-5734 (2006)

Populations of antigen-presenting cells (APCs) that are distinct from Langerhans cells have recently been identified in mouse skin. Now, a similar population of APCs has been found in human skin. Angel et al. describe a population of APCs that express intermediate levels of $\mathrm{CD} \mathrm{a}^{+}$but not the Langerhanscell marker CD207 and that is located close to the lymphatic vessels in the upper layers of the dermis. They termed these cells CD1 $a^{+}$dermal APCs. This population could prime naive CD4 ${ }^{+}$ T cells, owing to expression of MHC class II and co-stimulatory molecules. In a similar manner to Langerhans cells, the CD1 ${ }^{+}$ dermal APCs expressed CC-chemokine receptor 7 (CCR7) and could migrate in response to CCR7 ligands, which are known to mediate the migration of cells from the skin to the draining lymph nodes.

\section{MACROPHAGES}

Carbohydrate-independent recognition of collagens by the macrophage mannose receptor.

Martinez-Pomares, L. et al. Eur. J. Immunol. 36, 1074-1082 (2006)

A hallmark of alternatively activated macrophages is expression of the mannose receptor, which belongs to a family of four endocytic receptors with a common domain structure. The mannose receptor has two known, distinct lectin (carbohydratebinding) activities, which are mediated by its cysteine-rich domain and multiple C-type lectin-like domains (CTLDs). Located between these two domains is the fibronectin type II (FNII) domain, which is conserved among all mannose-receptor-family members and is important for collagen binding. MartinezPomares et al. examined whether the mouse mannose receptor could bind collagen, or if the CTLD- and cysteine-rich-domainmediated lectin activity was favoured. They found that the mannose receptor could indeed recognize and internalize collagen in a carbohydrate-independent manner, and that the FNII domain mediated this function. This paper describes a third distinct ligand-binding site for the mannose receptor and therefore has important implications for the study of this receptor at the molecular level.

\title{
A new way to get complement
}

Serum complement proteins comprise an important system that is responsible for several innate and adaptive immune defence mechanisms. There were three welldescribed pathways known to lead to the generation of a C3 convertase, which catalyses the proteolysis of complement component C3, and leads to the formation of $\mathrm{C} 3$ opsonins (C3b, iC3b and C3d) that fix to bacteria. This is a pivotal step in the initiation of the complement cascade. Now, a report recently published in Cell has identified a new pathway for the activation of $\mathrm{C} 3$.

C3 convertase can be formed by the classical C1q- and immunoglobulin-dependent pathway, the alternative factor-B-dependent pathway and the soluble mannose-binding lectin pathway.

The spleen clears microorganisms from the blood and individuals lacking this organ are more susceptible to Streptococcus pneumoniae. Innate resistance to $S$. pneumoniae has previously been shown to involve complement components C3 and C4, however this resistance has only a partial requirement for mediators of these three pathways, such as immunoglobulin, factor B and mannose-binding lectin.

Specific intracellular adhesion molecule-grabbing nonintegrin (SIGN)-R1, is a C-type lectin that is expressed at high levels by spleen marginal-zone macrophages and lymph-node macrophages. SIGN-R1 has previously been shown to be the main receptor for bacterial dextrans, as well as for the capsular pneumococcal polysaccharide (CPS) of S. pneumoniae. The authors, therefore, examined the role of this receptor in the activation of complement.

Using a monoclonal antibody that selectively downregulates SIGN-R1 expression in vivo, the authors show that in response to S. pneumoniae or CPS, SIGN-R1 mediates the immediate proteolysis of C3 and fixation of C3 opsonins to S. pneumoniae or to marginal-zone

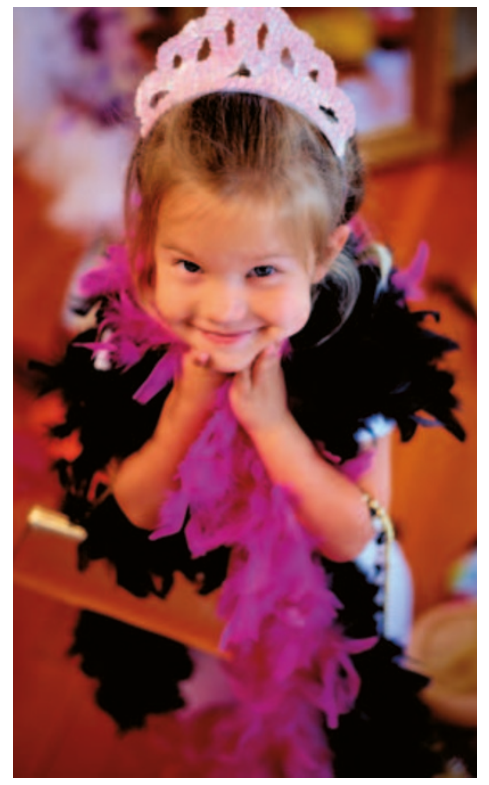

macrophages that had taken up CPS. These data indicate that SIGN-R1 is largely responsible for the rapid C3 convertase formation induced by S. pneumoniae in the spleen of mice. Interestingly, the authors showed that SIGN-R1 directly binds C1q and that $\mathrm{C} 3$ fixation by SIGN-R1 requires $\mathrm{C} 1 \mathrm{q}$ and $\mathrm{C} 4$ but not factor B or immunoglobulin.

The authors propose that in the SIGN-R1 pathway, after binding to polysaccharide, SIGN-R1 captures C1q. SIGN-R1 can then, in association with several other complement proteins including $\mathrm{C} 4$, lead to the formation of a C3 convertase and fixation of $\mathrm{C} 3$. Therefore, this new pathway for C3 fixation by SIGN-R1, which is unusual as it is a classical C1q-dependent pathway that does not require immunoglobulin, contributes to innate immune resistance to certain encapsulated microorganisms. In addition, these data provide an insight as to why the spleen is important for resistance to pneumococcal infection.

Olive Leavy

ORIGINAL RESEARCH PAPER Kang, Y.-S. et al. A dominant complement fixation pathway for pneumococcal polysaccharides initiated by SIGN-R1 interacting with C1q. Cell 125, 47-58 (2006) 